\title{
Cleft-Like Formation of the Aortic Valve in an Adult Patient with a Single Coronary Artery
}

\author{
Hiroaki Kawano ${ }^{1}$, Tomayoshi Hayashi ${ }^{2}$, Takako Minami ${ }^{1}$, \\ Yuji Koide ${ }^{1}$, Kiyoyuki Eishi ${ }^{3}$ and Koji Maemura ${ }^{1}$
}

\begin{abstract}
A 51-year-old woman was admitted to our hospital with heart failure due to aortic regurgitation. Examination showed severe aortic regurgitation mainly due to cleft-like deformity of the right coronary cusp and single coronary artery. Aortic valve replacement was performed, and the deformity was seen in all aortic cusps. Histological study showed elongation of the leaflets by myofibrotic and fibrotic hyperplasia without calcification and inflammation in the aortic valve. This deformity likely arose from an acquired modification of a congenitally malformed aortic valve.
\end{abstract}

Key words: aortic valve, aortic regurgitation, pathology, single coronary artery

(Intern Med 51: 2341-2345, 2012)

(DOI: 10.2169/internalmedicine.51.7808)

\section{Introduction}

Aortic regurgitation (AR) can arise due to abnormalities in the aortic leaflets (e.g., infective endocarditis, congenital bicuspid valve, rheumatic valve, floppy valve), the aortic root and annulus (e.g., syphilis, Marfan's dissection), or both (e.g., ankylosing spondylitis), and from disease affecting neither the aorta nor the valve (e.g., ventricular septal defect, systemic hypertension) (1-9). Abnormalities of the aortic cusps leading to AR include congenital leaflet abnormalities, such as bicuspid, unicuspid, or quadricuspid valves, or rupture of a congenitally fenestrated valve (9). The present report describes a case of cleft-like formation of the tricuspid aortic valve in a patient with AR and a left single coronary artery.

\section{Case Report}

A 51-year-old woman was admitted to our hospital with heart failure due to aortic regurgitation. She had no past history of rheumatic fever. Heart murmur was documented at the age of 17 , and she was diagnosed with asymptomatic aortic regurgitation (Seller's grade III) by aortography at our hospital at the age of 22. Since then, she underwent routine follow-up at our hospital every 3 months. She developed chest discomfort at the age of 38 , and a transthoracic echo cardiography (TTE) showed a left ventricular ejection fraction (LVEF) of 74\%, LV end-diastolic dimension (LVDD) of $54 \mathrm{~mm}$, aortic dimension of $32 \mathrm{~mm}$, and moderate AR. At the age of 44, aortic valve replacement was recommended due to New York Heart Association classification status II with LVDD of $55 \mathrm{~mm}$ and moderate AR by TTE, but the patient declined. Her cardiomegaly gradually progressed, and she ultimately agreed to undergo aortic valve replacement at the age of 51 .

On admission, physical examination revealed a height of $146 \mathrm{~cm}$, a weight of $48.2 \mathrm{~kg}$, blood pressure of 104/50 $\mathrm{mmHg}$, and a regular pulse rate of 60 beats/min. A Levine grade III diastolic murmur was heard at 3 left sternal border, and a Levine grade II systolic murmur was heard at 2 right sternal border, but the lung fields were clear to auscultation, and she had no peripheral edema. Only mild cardiomegaly was present on chest X-ray, and the electrocardiogram (ECG) showed sinus rhythm and mild left ventricular hypertrophy on admission.

\footnotetext{
${ }^{1}$ Department of Cardiovascular Medicine, Nagasaki University Graduate School of Biomedical Sciences, Japan, ${ }^{2}$ Department of Pathology, Nagasaki University Hospital, Japan and ${ }^{3}$ Department of Cardiovascular Surgery, Nagasaki University Graduate School of Biomedical Sciences, Japan

Received for publication March 26, 2012; Accepted for publication May 23, 2012

Correspondence to Dr. Hiroaki Kawano, hkawano@nagasaki-u.ac.jp
} 

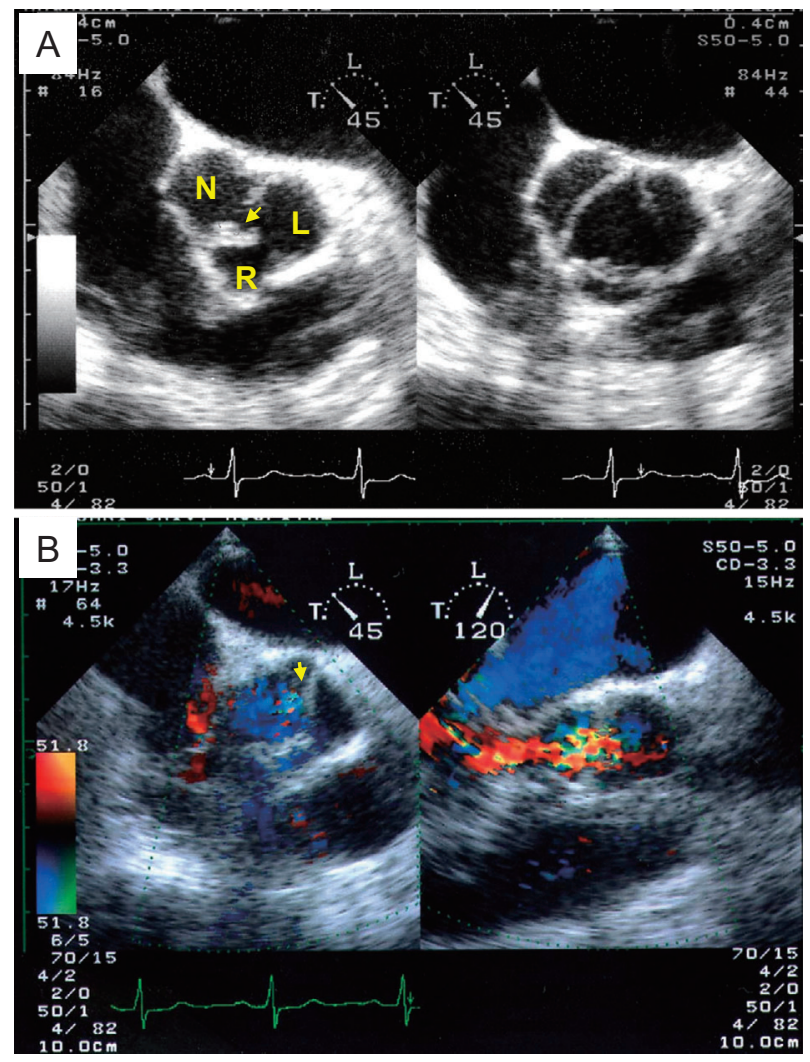

Figure 1. Transesophageal echocardiography showed that the right coronary cusp with thickened free edge was mildly hypoplastic and that there was a gap in the center of the aortic valve orifice (A). Aortic regurgitant jet came from the central space made by three coronary cusps (B). N: non-coronary cusp, R: right coronary cusp, L: left coronary cusp

Laboratory testing showed a white blood cell count of $6,000 / \mathrm{mm}^{3}$, blood urea nitrogen of $6 \mathrm{mg} / \mathrm{dL}$, creatinine of $0.5 \mathrm{mg} / \mathrm{dL}$, aspartate aminotransferase of $23 \mathrm{IU} / \mathrm{L}$, alanine aminotransferase of $22 \mathrm{IU} / \mathrm{L}$, lactate dehydrogenase of 276 IU/L, creatine kinase of $139 \mathrm{IU} / \mathrm{L}$, and C-reactive protein of $0.03 \mathrm{mg} / \mathrm{dL}$.

A TTE demonstrated a LVEF of $63 \%$, LVDD of $59 \mathrm{~mm}$, aortic dimension of $33 \mathrm{~mm}$, and severe AR with mild mitral regurgitation. Transesophageal echocardiography showed that the right coronary cusp (RCC) was mildly hypoplastic and that the AR jet came from the central space made by three coronary cusps (Fig. 1). Coronary angiogram (CAG) showed a single coronary artery originating from the left coronary cusp (LCC) and a right coronary artery branching from the left ascending coronary artery and running to the anterior wall of right ventricle (Fig. 2).

Aortic valve replacement was performed. The orifice of the right coronary artery was not detected at operation. Macroscopic examination of the aortic valve revealed that the aortic valve was tricuspid and that the right coronary cusp was smaller than the other cusps. Further, all the cusps were deformed: 1) a cleft-like formation was seen at the middle of the free edge especially in the RCC, 2) the left coronary cusp had a v-shaped depression at the middle of the free edge, and 3) the non-coronary cusp had a wide depression at the middle of the free edge (Fig. 3A).

Histological examination revealed: 1) aortic valves with normal components (i.e., proximalis, spongiosa, and distalis) (Fig. 3C), 2) fibrous thickening and elongation of the anatomical edge of these cusps except for the middle of the anatomical edge of the cusps, which caused cleft-like formation (Fig. 3C, 4A), and 3), laminar formation of many myofibrocytes or myofibroblasts positive for $\alpha$-smooth muscle actin in the elongated area of the cusps (Fig. 4B), and several fibroblasts at the tip of the leaflet were positive for $\mathrm{Ki}$ 67, a cell proliferation marker (Fig. 4C).

\section{Discussion}

One previous report describes a case of cleft of the aortic valve (10), in which a cleft-like deformity was formed by the fused portion of cusps in a patient with bicuspid aortic valve. A congenital bicuspid aortic valve is present in about $1 \%$ to $2 \%$ of the population. Although it develops severe $\mathrm{AR}$, most patients with it develop severe aortic stenosis due to calcification after 50 years of age (11). The present case had a cleft-like formation of the tricuspid aortic valve because there was no fusion of the cusps confirmed by surgical and macroscopic inspection, and she had a single coronary artery. An isolated single coronary artery is a rare congenital anomaly, occurring in approximately $0.024 \%$ of the population $(12,13)$, and approximately one-third of cases with a single coronary artery have other cardiovascular anomalies (14). There are five case reports of patients with a single coronary artery and AR (15-19), and four of these five cases had other congenital anomalies (i.e., bicuspid aortic valve, ventricular septal defect, or coronary aneurysms) (16-19). Although there was only one adult case report of a single coronary artery and AR without other congenital anomalies, the cause of AR was not determined or described for that patient. Moreover, there have been no published reports of a cleft-like aortic valve anomaly in a patient with a single coronary artery in the absence of other congenital anomalies.

In the present case, all three aortic leaflets were deformed without calcification, and histologic evidence of inflammation was not present. This suggests that deformation of the aortic valve was related to congenital malformation. Moreover, immunohistochemical staining showed that ongoing proliferation of fibroblasts leading to elongation of the free edge of the leaflet, resulted in a cleft-like formation. Taken together, these observations suggest that this deformity likely resulted from adaptive modification of a congenitally malformed aortic valve.

The mechanism of elongation of the aortic valve is unclear. Previous studies suggested that dilatation of the aortic root (20) or a non-compliant aortic root (21) can lead to enlargement or elongation of leaflets, which may render leaflets more susceptible to mechanical stress. Thus, aortic valve enlargement or elongation may represent reactive compensa- 


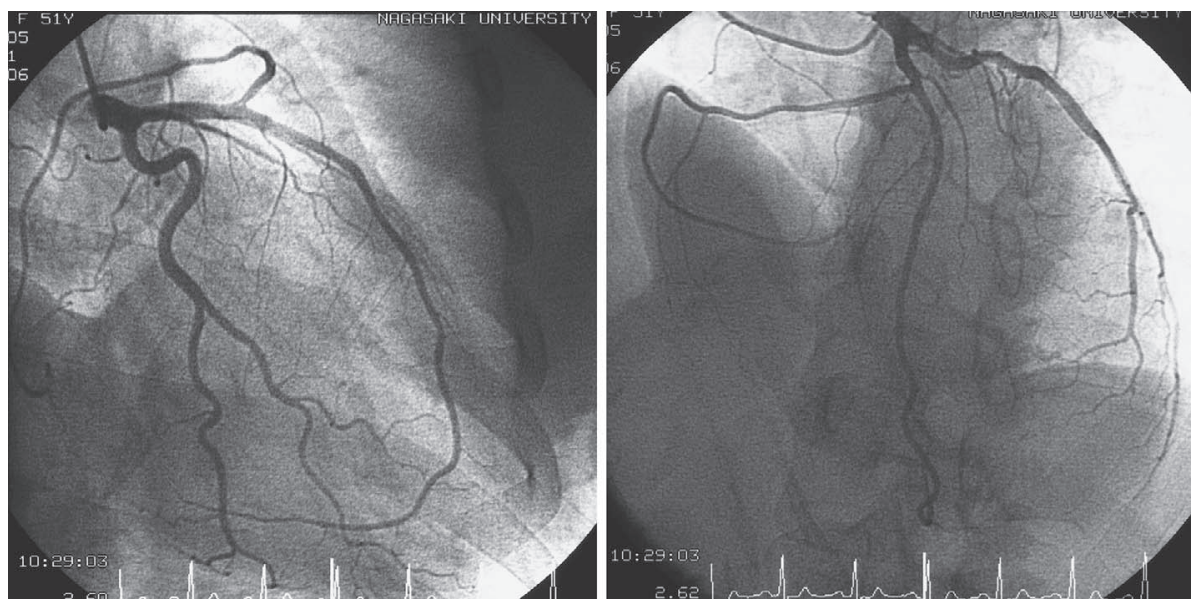

Figure 2. Coronary angiogram showed a single coronary artery originating from the left coronary cusp and a right coronary artery branching from the left ascending coronary artery and running to the anterior wall of the right ventricle.

123
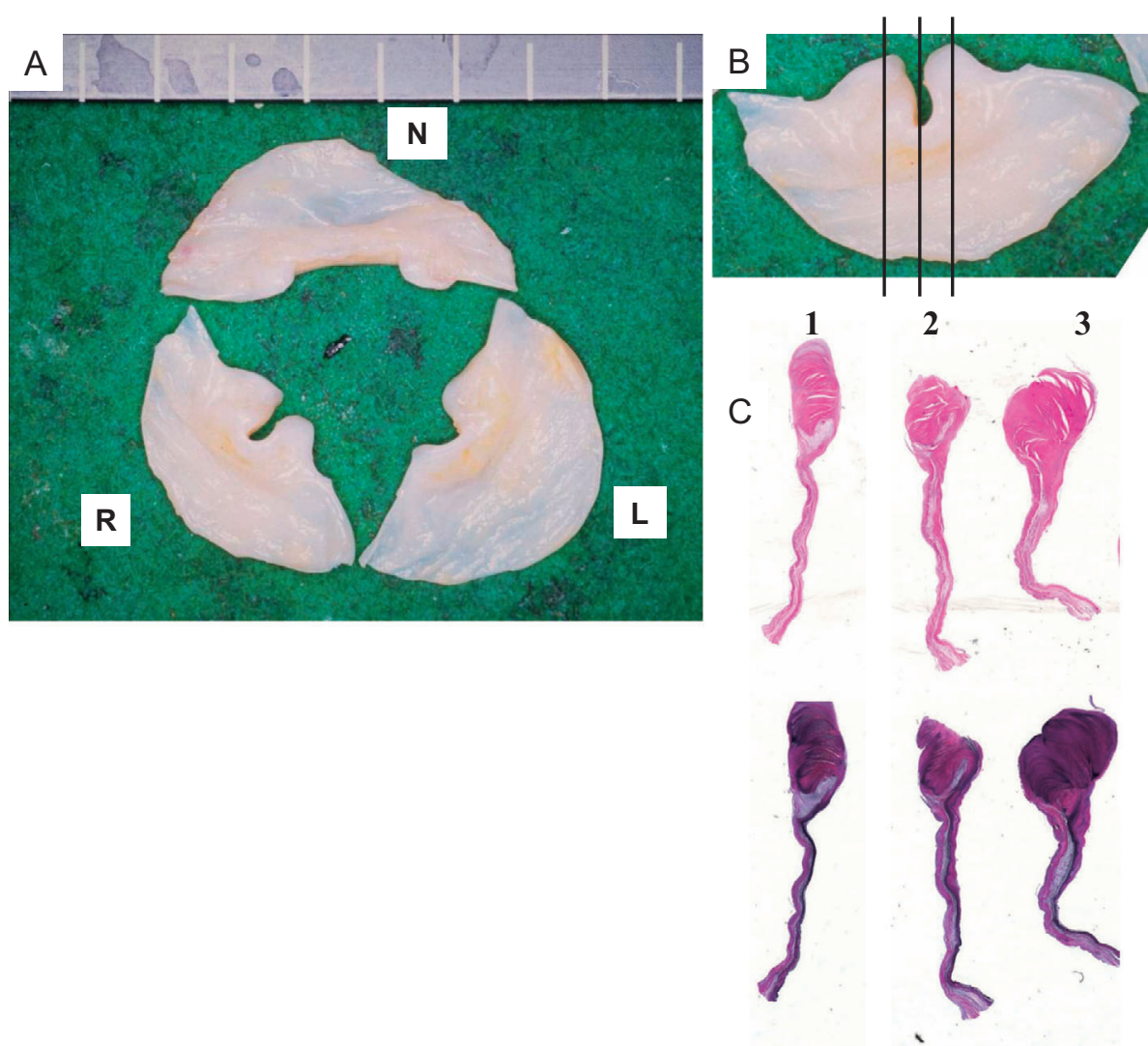

Figure 3. (A) Gross appearance of the aortic valve removed at surgery. A cleft-like formation was seen at the middle of the free edge especially in the right coronary cusp. The left coronary cusp had a v-shaped depression at the middle of the free edge, and the non-coronary cusp had a wide depression at the middle of the free edge. (B and $\mathrm{C}$ ) Histological examination revealed that the edges of the aortic leaflet at both sides ( 1 and 3 ), but not the center of the aortic leaflet, were thickened and elongated by fibrotic hyperplasia (2) and that aortic valves had normal components i.e., proximalis, spongiosa, and distalis (Hematoxylin and Eosin staining $\times 2$, Weigert Van Gieson stain $\times 2$ ).

tions in an effort to maintain cusp coaptation for the prevention of aortic regurgitation. Although the present case did not have significant aortic root dilatation, the smaller right coronary cusp (relative to other cusps) combined with congenital deformity of aortic valves resulted in a gap in the center of aortic valve when the aortic valve was in the 


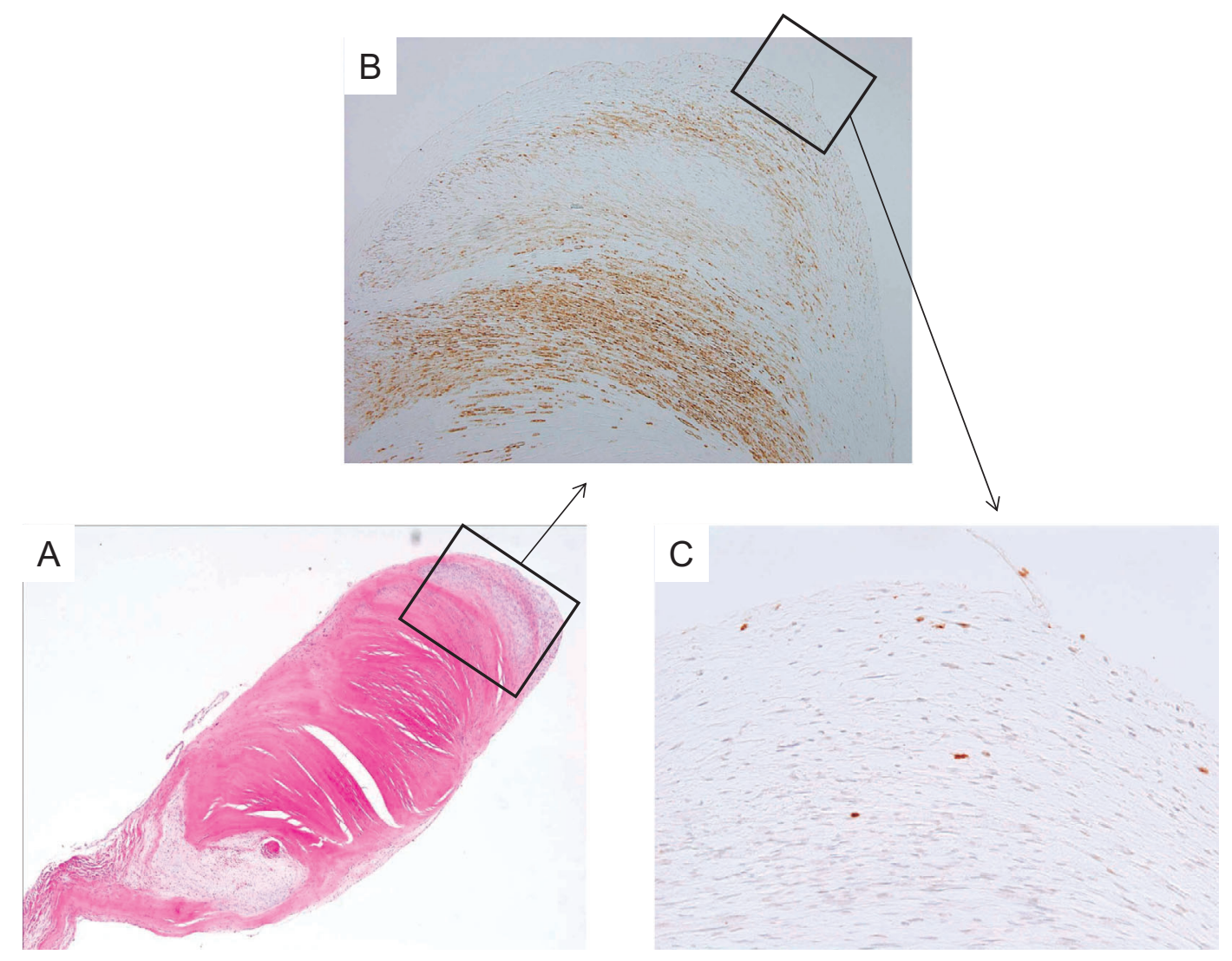

Figure 4. Fibrous thickening and elongation of the anatomical edge of these cusps, except for the middle of the anatomical edge of the cusps, was seen (A, Hematoxylin and Eosin staining $\times 20$ ), and laminar formation of many myofibrocytes or myofibroblasts positive for $\alpha$-smooth muscle actin was seen in the elongated area of cusps $(B, \times 40)$. Several fibroblasts at the tip of leaflet were positive for $\mathrm{Ki67}$, a cell proliferation marker $(\mathrm{C}, \times 100)$.

closed position. Thus, these situations may induce mechanical stress, thereby producing aortic valve elongation. This reactive compensation may be related to the slow progression of AR in the present case because AR severity and LVDD did not change for about 30 years. In the present case, the center of the leaflet did not elongate, and there was a fibrous thickened nodule ("Arantius nodule") in the center of the free edge of the normal aortic leaflet. Thus, an Arantius nodule may have prevented elongation of the edge of the aortic leaflet in the present case.

In conclusion, the presence of tricuspid aortic valve malformation as well as other congenital anomalies should be considered in patients with a single coronary artery and aortic regurgitation.

\section{The authors state that they have no Conflict of Interest (COI).}

\section{References}

1. Waller BF, Howard J, Fess S. Pathology of aortic valve stenosis and pure aortic regurgitation: a clinical morphologic assessment: Part II. Clin Cardiol 17: 150-156, 1994.

2. Roberts WC, Kehoe JA, Carpenter DF, Golden A. Cardiac valvular lesions in rheumatoid arthritis. Arch Intern Med 122: 141-146, 1968.
3. Oh WM, Taylor RT, Olsen GJ. Aortic regurgitation in systemic lupus erythematosus requiring aortic valve replacement. Br Heart J 36: 413-416, 1974.

4. Allen WM, Matloff JM, Fishbein MC. Myxoid degeneration of the aortic valve and isolated severe aortic regurgitation. Am J Cardiol 55: 439-444, 1985.

5. Fenoglio JJ Jr, McAllister HA, DeCastro CM, Davia JE, Cheitlin MD. Congenital bicuspid aortic valve after age 20. Am J Cardiol 39: 164-169, 1977.

6. Roberts WC, Morrow AG, McIntosh CL, Jones M, Epstein SE. Congenitally bicuspid aortic valve causing severe, pure aortic regurgitation without superimposed infective endocarditis: analysis of 13 patients requiring aortic valve replacement. Am J Cardiol 47: 206-209, 1981

7. Olson LJ, Subramanian R, Edwards WD. Surgical pathology of pure aortic insufficiency: a study of 225 cases. Mayo Clin Proc 59: 835-841, 1984.

8. Roberts WC, Ko JM, Moore TR, Jones WH III. Causes of pure aortic regurgitation in patients having isolated aortic valve replacement at a single US tertiary hospital (1993 to 2005). Circulation 114: 422-429, 2006.

9. Maganti K, Rigolin VH, Sarano ME, Bonow RO. Valvular heart disease: diagnosis and management. Mayo Clin Proc 85: 483-500, 2010.

10. Mancuso D, Basso C, Cardaioli C, Cardaioli P, Thiene G. Clefted bicuspid aortic valve. Cardiovasc Pathol 11: 217-220, 2002.

11. Otto CM, Bonow RO. Valvular heart disease. In: Brawnwald's Heart Disease. 9th ed. Bonow RO, Mann DL, Zipes DP, Libby P, Brawnwald E, Eds. Elsevier Saunders, Philadelphia, 2012: 1468- 
1539.

12. Sharbaugh AH, White RS. Single coronary artery. Analysis of the anatomic variation, clinical importance, and report of five cases. JAMA 230: 243-246, 1974.

13. Lipton MJ, Barry WH, Obrez I, Silverman JF, Wexler L. Isolated single coronary artery: diagnosis, angiographic classification, and clinical significance. Radiology 130: 39-47, 1979.

14. Ogden JA, Goodyer AV. Patterns of distribution of the single coronary artery. Yale J Biol Med 43: 11-21, 1970.

15. Katsetos MC, Toce DT. Single coronary artery with aortic regurgitation. Cardiovasc Intervent Radiol 26: 567-578, 2003.

16. Nanba M, Hanawa N, Mita H, Hongo M, Minemawari M. A case of single coronary artery complicated with bicuspid aortic valve and aortic regurgitation. Nippon Naika Gakkai Zasshi 80: 19591961, 1991 (in Japanese).

17. Shore DF, Ho SY, Anderson RH, de Leval M, Lincoln C. Aorto- pulmonary septal defect coexisting with ventricular septal defect and pulmonary atresia. Ann Thorac Surg 35: 132-137, 1983.

18. Goto Y, Matsuno Y, Yoshikane H, et al. Ruptured aneurysm of the sinus Valsalva with single coronary artery. Cathet Cardiovasc Diagn 17: 172-174, 1989.

19. Oohara K, Yamazaki T, Sakaguchi K, Nakayama M, Kobayashi A. Acute aortic dissection, aortic insufficiency, and a single coronary artery in a patient with Turner's syndrome. J Cardiovasc Surg 36: 273-275, 1995.

20. Thubrikar MJ, Labrosse MR, Zehr KJ, Robicsek F, Gong GG, Fowler BL. Aortic root dilatation may alter the dimensions of the valve leaflets. Eur Cardiothorac Surg 28: 850-855, 2005.

21. Fokin AA, Robicsek F, Cook JW, Thubrikar MJ, Schaper J. Morphological changes of the aortic valve leaflets in non-compliant aortic roots: in-vivo experiments. J Heart Valve Dis 13: 444-451, 2004.

(C) 2012 The Japanese Society of Internal Medicine http://www.naika.or.jp/imonline/index.html 